\title{
Bilateral pneumothoraces and pleural effusions complicating rheumatoid lung disease
}

\author{
O AYZENBERG, DB REIFF, L LEVIN
}

From the Department of Medicine, Groote Schuur Hospital, Observatory, South Africa

\begin{abstract}
While pleural-pulmonary lesions are not uncommon in rheumatoid disease, pneumothoraces are rare. We describe a case of bilateral pneumothoraces and chyliform effusions with histological evidence of rheumatoid lung disease.
\end{abstract}

\section{Case report}

A 58-year-old man was referred to Groote Schuur Hospital from a peripheral hospital and in October 1980 was found to have a pneumothorax on the left side. In June 1981 he presented to the same hospital complaining of progressive shortness of breath; a left pneumothorax was again found and drained. He continued to complain of shortness of breath, and a chest radiograph in September 1981 showed recurrence of the left pneumothorax and he was referred for further investigation. He was a bricklayer but had stopped working because of considerable deformities of his joints. There was a history of smoking two to three cigarettes daily but he had had no exposure to mineral dust. Over the past three years he had developed progressive deformity of his joints associated with some morning stiffness but no dry eyes or mouth.

Physical examination showed typical rheumatoid deformities of the interphalangeal, metacarpophalangeal, and wrist joints with interosseus muscle wasting and fixed deformities of the ankles. In addition, there were large non-tender periarticular nodules on the extensor surface of both elbows. On examination of the chest, motion was not limited but there was dullness to percussion with decreased air entry and vocal resonance at both bases. The remainder of the findings in the physical examination were normal.

The chest radiograph showed a normal-sized heart, bilateral pneumothoraces, and pleural effusions with appreciable pleural thickening (fig). The electrocardiogram and echocardiogram were normal. The erythrocyte sedimentation rate was $26 \mathrm{~mm}$ in the first hour, haemoglobin $14.1 \mathrm{~g} / \mathrm{dl}$, red cell count 5.44 , white cell count $12.9 \times$ $10 \%$ (79\% polymorphs, $15 \%$ lymphocytes, $3 \%$ monocytes, $2 \%$ eosinophils). Serum calcium, phosphate, and urate concentrations were normal. The serum complement concentration was 169 units/ml (normal 160-220 units/ml). In investigations for rheumatoid factor he gave a negative result in the sheep-cell agglutination test but was latex positive to a titre of 80 . All other investigations gave normal results.

Address for reprint requests: Dr O Ayzenberg, Department of Medicine, Groote Schuur Hospital, Observatory, Cape 7925, South Africa.

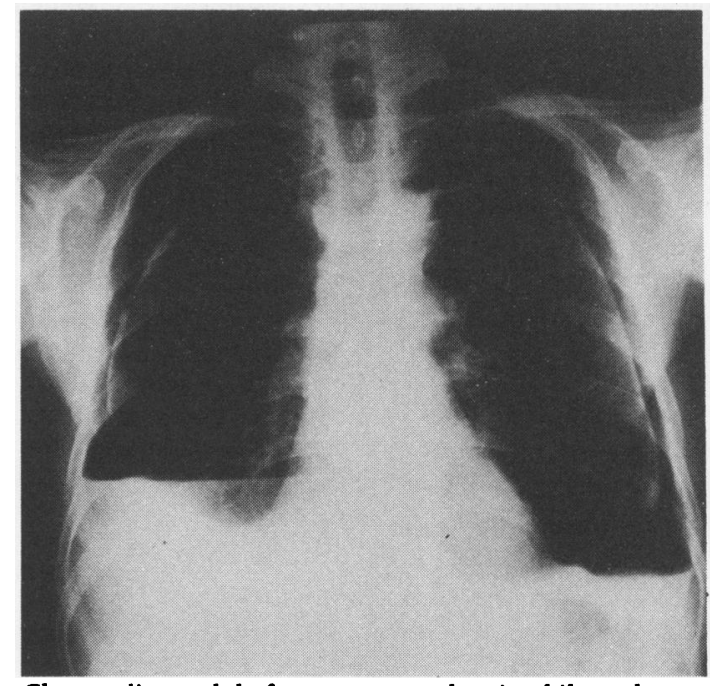

Chest radiograph before treatment showing bilateral pneumothoraces and pleural effusions.

Bilateral chest drains were inserted and a litre of chyliform fluid was aspirated. This showed many cholesterol crystals, with a lactate dehydrogenase (LDH) levels of 4440 units, glucose concentration $0.3 \mathrm{mmol} / 1(5.4 \mathrm{mg} /$ $100 \mathrm{ml}$ ) and cholesterol $9 \cdot 1 \mathrm{mmol} / 1(351 \mathrm{mg} / 100 \mathrm{ml})$. There were no acid-fast bacilli and culture for tubercle bacilli gave negative results. A nodule excised from the right elbow showed granulomas with central necrosis consistent with a rheumatoid nodule. Despite application of suction to the drain the lung did not re-expand. The patient was referred for bilateral pleurectomy, which was done in two stages, and his progress has since been satisfactory. The pleural biopsy specimen showed thickened fibrotic tissue and the surface was lined by a layer of fibrinoid hyaline material. There were focal lymphocyte collections and occasional granulomas showing central necrosis with peripheral palisading of epithelioid cells. A lung biopsy done at operation showed patchy interstitial fibrosis compatible with rheumatoid lung disease.

\section{Discussion}

Although pleural effusion is a not uncommon feature of rheumatoid lung disease pneumothorax is rare. In one series effusion occurred in $37 \%$ of patients with 
rheumatoid lung disease whereas pneumothorax occurred in only $6 \% .^{1}$ Our patient presented with bilateral pneumothoraces and pleural effusions and this is the first documentation of the simultaneous occurrence of these complications.

The presence of a high cholesterol content in the pleural fluid from these patients has also been noted. ${ }^{2}$ There is no satisfactory explanation for the chyliform pleural fluid, although some authors have postulated that it is due to the longstanding accumulation of a breakdown product of lymphocytes, which is not reabsorbed because of gross pleural thickening. The high LDH and low glucose content of the pleural fluid found in our patient is also characteristic of pleural effusion complicating rheumatoid lung disease.
Pneumothorax is thought to be due to cavitation of $\overrightarrow{\overline{\vec{N}}}$ rheumatoid nodule which perforates into the pleural space, creating a continuous leak. It therefore almost alwas requires surgical intervention. ${ }^{3}$

\section{References}

${ }^{1}$ Martell W, Abell MR, Mikkelsen WM, Whitehouse WM. Pulmonary and pleural lesions in rheumatoid disease. Radiology 1968;90:641-52.

${ }^{2}$ Ferguson GC. Cholesterol pleural effusions rheumatoid lung disease. Thorax 1966;21:577-82. $\times$

${ }^{3}$ Hingle W, Yates DAH. Pyopneumothorax complicatifg rheumatoid lung disease. Ann Rheum Lis $1965 ; 24: 57-60$. 